\title{
PATH PROPERTIES OF THE PRIMITIVES OF A BROWNIAN MOTION
}

\author{
ZHENGYAN LIN
}

(Received 16 June 1999; revised 28 June 2000)

Communicated by V. Stefanov

\section{Abstract}

Let $\{W(t), t \geq 0\}$ be a standard Brownian motion. For a positive integer $m$, define a Gaussian process

$$
X_{m}(t)=\frac{1}{m !} \int_{0}^{t}(t-s)^{m} d W(s)
$$

Watanabe and Lachal gave some asymptotic properties of the process $X_{m}(\cdot), m \geq 1$. In this paper, we study the bounds of its moduli of continuity and large increments by establishing large deviation results.

2000 Mathematics subject classification: primary 60F15, 60J65, 60G15; secondary 60G17.

Keywords and phrases: moduli of continuity, large increments, Brownian motion, primitive.

\section{Introduction}

Let $\{W(t), t \geq 0\}$ be a standard Brownian motion. For a positive integer $m$, define a Gaussian process

$$
X_{m}(t)=\frac{1}{m !} \int_{0}^{t}(t-s)^{m} d W(s),
$$

which was first mentioned by Shepp [4]. This class of processes arises in several domains of applied mathematics. For instance, the process $X_{1}(\cdot)$, which has been studied at length, is the solution of Langevin's equation under certain physical conditions. Wahba $[5,6]$ used $X_{n}(\cdot)$ to derive a correspondence between smoothing by splines and Bayesian estimation in certain stochastic models.

Watanabe [7] established a law of the iterated logarithm for $X_{1}(\cdot)$ (in fact, his result concerns a larger class of Gaussian processes). Lachal $[2,3]$ studied the law of the

(C) 2001 Australian Mathematical Society 0263-6115/2001 \$A2.00+0.00 
iterated logarithm and regular points for $X_{m}(\cdot), m \geq 1$. Moreover, Lachal [2] obtained some integral tests that precisely characterize the upper functions for $X_{m}$, which is an important result in the asymptotic study of $X_{m}$.

In this paper we study path behaviour of the process $X_{m}(\cdot)$. By establishing results on large deviations, we investigate the moduli of continuity and large increment properties for $X_{m}(\cdot), m \geq 1$, and give their upper and lower bounds. Note that increments of $X_{m}(\cdot)$ are neither independent nor stationary, moreover $X_{m}(\cdot)$ is also not a stationary process. Usually, stationarity of increments is required for investigating the moduli of continuity and large increments of a process.

First of all, we give some moment results. We have

$$
E X_{m}^{2}(t)=\frac{1}{(m !)^{2}} \int_{0}^{t}(t-s)^{2 m} d s=: b_{m} t^{2 m+1},
$$

where $b_{m}=(m !)^{-2}(2 m+1)^{-1}$, and for any $h>0$

$$
\begin{aligned}
& E\left(X_{m}(t+h)-X_{m}(t)\right)^{2} \\
&= \frac{1}{(m !)^{2}} E\left(\int_{0}^{t+h}(t+h-s)^{m} d W(s)-\int_{0}^{t}(t-s)^{m} d W(s)\right)^{2} \\
&= \frac{1}{(m !)^{2}}\left\{E\left(\int_{0}^{t}\left(\sum_{j=1}^{m}\left(\begin{array}{c}
m \\
j
\end{array}\right)(t-s)^{m-j} h^{j}\right) d W(s)\right)^{2}\right. \\
&\left.+E\left(\int_{t}^{t+h}(t+h-s)^{m} d W(s)\right)^{2}\right\} \\
&=: \sum_{j=2}^{2 m+1} b_{m j} h^{j} t^{2 m+1-j}
\end{aligned}
$$

for some positive $b_{m j}, j=2, \ldots, 2 m+1$, where $b_{m 2}=((m-1) !)^{-2}(2 m-1)^{-1}$. Equality (1.3) implies

$$
E\left(X_{m}(t+h)-X_{m}(t)\right)^{2}=(1+\delta(h / t)) b_{m 2} h^{2} t^{2 m-1},
$$

where $0<\delta(x) \rightarrow 0$ as $x \rightarrow 0$. Hence

$$
\begin{aligned}
E\left(X_{m}(t+h) X_{m}(t)\right) & =\frac{1}{2} E\left\{X_{m}^{2}(t+h)+X_{m}^{2}(t)-\left(X_{m}(t+h)-X_{m}(t)\right)^{2}\right\} \\
& =\frac{1}{2} b_{m}\left((t+h)^{2 m+1}+t^{2 m+1}\right)-\frac{1}{2} \sum_{j=2}^{2 m+1} b_{m j} h^{j} t^{2 m+1-j}
\end{aligned}
$$

Put $Y_{m}(t)=X_{m}(t) / t^{m-1 / 2}$. By $(1.2)$

$$
E Y_{m}^{2}(t)=b_{m} t^{2}
$$


Using (1.2), (1.3) and (1.5) we have

$$
\begin{aligned}
& E\left(Y_{m}(t+h)-Y_{m}(t)\right)^{2} \\
&= E\left\{\frac{X_{m}(t+h)-X_{m}(t)}{(t+h)^{m-1 / 2}}-\left(\frac{1}{t^{m-1 / 2}}-\frac{1}{(t+h)^{m-1 / 2}}\right) X_{m}(t)\right\}^{2} \\
&= \frac{\sum_{j=2}^{2 m+1} b_{m j} h^{j} t^{2 m+1-j}}{(t+h)^{2 m-1}}+\frac{\left((t+h)^{m-1 / 2}-t^{m-1 / 2}\right)^{2}}{t^{2 m-1}(t+h)^{2 m-1}} b_{m} t^{2 m+1} \\
&-\frac{2\left((t+h)^{m-1 / 2}-t^{m-1 / 2}\right)}{t^{m-1 / 2}(t+h)^{2 m-1}}\left\{\frac{1}{2} b_{m}\left((t+h)^{2 m+1}-t^{2 m+1}\right)\right. \\
&\left.-\frac{1}{2} \sum_{j=2}^{2 m+1} b_{m j} h^{j} t^{2 m+1-j}\right\} \\
&=: B_{m} h^{2}+g_{m}(h, t),
\end{aligned}
$$

where

$$
\begin{gathered}
B_{m}=b_{m 2}+b_{m}\left\{\left(m-\frac{1}{2}\right)^{2}-\left(m-\frac{1}{2}\right)(2 m+1)\right\} \\
=b_{m 2}-b_{m}\left(m-\frac{1}{2}\right)\left(m+\frac{3}{2}\right), \\
g_{m}(h, t)=O\left(h^{3} t\right) \quad \text { as } h t \rightarrow 0,
\end{gathered}
$$

which implies that

$$
E\left(Y_{m}(t+h)-Y_{m}(t)\right)^{2}=(1+o(1)) B_{m} h^{2} \quad \text { as } h t \rightarrow 0 .
$$

\section{Large deviations}

First we quote a well-known lemma.

LEMMA 2.1 (Fernique). Let $G(t)$ be a Gaussian process on $[0,1]$ with $E G^{2}(t) \leq$ $A^{2}$ and $E(G(t)-G(s))^{2} \leq \sigma^{2}(|t-s|)$, where $\sigma(\cdot)$ is a continuous nondecreasing function satisfying

$$
\int_{1}^{\infty} \sigma\left(e^{-x^{2}}\right) d x<\infty
$$

Then, for $x \geq 2$, we have

$$
P\left\{\sup _{0 \leq t \leq 1}|G(t)| \geq x\left(A+\int_{1}^{\infty} \sigma\left(e^{-y^{2}}\right) d y\right)\right\} \leq c e^{-x^{2} / 2},
$$

where $c$ is an absolute constant. 
The following is a large deviation result for small time increments.

PROPOSITION 2.1. For any $\varepsilon>0$, there exist positive numbers $h_{0}, x_{0}, c_{1}$ and $C_{1}$ such that for any $0<h \leq h_{0}$ and $x \geq x_{0}$

$$
P\left\{\sup _{0<t \leq 1-h \sup _{0 \leq s \leq h}} \frac{\left|X_{m}(t+s)-X_{m}(t)\right|}{(t \vee h)^{m-1 / 2}} \geq(1+\varepsilon) b_{m 2}^{1 / 2} h x\right\} \leq C_{1}\left(e^{-c_{1} x^{2}}+h^{-1} e^{-x^{2} / 2}\right) .
$$

PROOF. For any $t>0$ and integer $r>0$, let $t_{r}=\left[t 2^{r} / h\right] /(2 r / h)$, and write, for $r h<1-h$,

$$
\begin{aligned}
\sup _{0<t \leq 1-h} & \sup _{0 \leq s \leq h} \frac{\left|X_{m}(t+s)-X_{m}(t)\right|}{(t \vee h)^{m-1 / 2}} \\
= & \sup _{0<t \leq r h} \sup _{0 \leq s \leq h} \frac{\left|X_{m}(t+s)-X_{m}(t)\right|}{(t \vee h)^{m-1 / 2}} \vee \sup _{r h \leq t \leq 1-h} \sup _{0 \leq s \leq h} \frac{\left|X_{m}(t+s)-X_{m}(t)\right|}{t^{m-1 / 2}} \\
= & : I_{1} \vee I_{2} .
\end{aligned}
$$

Noting $t \vee h \geq(t+h) / 2$, we have

$$
I_{1} \leq 2^{m-1 / 2} \sup _{0<t \leq r h} \sup _{0 \leq s \leq h} \frac{\left|X_{m}(t+s)-X_{m}(t)\right|}{(t+h)^{m-1 / 2}} \leq 2^{m+1 / 2} \sup _{0<t \leq(1+r) h}\left|Y_{m}(t)\right| .
$$

Let $Z_{m}(t)=Y_{m}((1+r) h t), 0<t \leq 1$. We will use Lemma 2.1 with $A=b_{m}^{1 / 2}(1+r) h$ and $\sigma(s)=\left(2 B_{m}\right)^{1 / 2}(1+r) h s$. Put $D=(1+r)\left(b_{m}^{1 / 2}+\left(2 B_{m}\right)^{1 / 2} \int_{1}^{\infty} e^{-y^{2}} d y\right)$. For any given $\varepsilon>0$, take $r=r(\varepsilon)$ to be specified later on. By Lemma 2.1, we have

$$
P\left\{I_{1} \geq b_{m 2}^{1 / 2} h x\right\} \leq P\left\{\sup _{0<t \leq 1}\left|Z_{m}(t)\right| \geq\left(b_{m 2}^{1 / 2} 2^{-(m+1 / 2)} D^{-1}\right) D h x\right\} \leq C e^{-c_{1} x^{2}}
$$

for $x \geq x_{0}:=b_{m 2}^{-1 / 2} 2^{m+1 / 2} D$, where $c_{1}=b_{m 2} 2^{-(2 m+1)} D^{-2} / 2$.

Consider $I_{2}$ now. We shall use a method similar to that in [1]. For $r h<t \leq 1-h$, $0 \leq s \leq h$, which implies that

$$
\frac{1}{t^{m-1 / 2}} \leq\left(1+\frac{1}{r}\right)^{m-1 / 2} \frac{1}{(t+s)^{m-1 / 2}} \leq\left(1+\frac{1}{r}\right)^{m-1 / 2} \frac{1}{(t+s)_{r+j+1}^{m-1 / 2}}
$$

for any $j \geq 0$, we have

$$
\begin{aligned}
& \frac{\left|X_{m}(t+s)-X_{m}(t)\right|}{t^{m-1 / 2}} \\
& \quad \leq \frac{\left|X_{m}\left((t+s)_{r}\right)-X_{m}\left(t_{r}\right)\right|}{t^{m-1 / 2}}+\frac{\left|X_{m}\left((t+s)_{r}\right)-X_{m}(t+s)\right|}{t^{m-1 / 2}}
\end{aligned}
$$




$$
\begin{aligned}
& +\frac{\left|X_{m}\left(t_{r}\right)-X_{m}(t)\right|}{t^{m-1 / 2}} \\
\leq & \frac{\left|X_{m}\left((t+s)_{r}\right)-X_{m}\left(t_{r}\right)\right|}{t^{m-1 / 2}}+\sum_{j=0}^{\infty} \frac{\left|X_{m}\left((t+s)_{r+j+1}\right)-X_{m}\left((t+s)_{r+j}\right)\right|}{t^{m-1 / 2}} \\
& +\sum_{j=0}^{\infty} \frac{\left|X_{m}\left(t_{r+j+1}\right)-X_{m}\left(t_{r+j}\right)\right|}{t^{m-1 / 2}} \\
\leq & \frac{\left|X_{m}\left((t+s)_{r}\right)-X_{m}\left(t_{r}\right)\right|}{t_{r}^{m-1 / 2}} \\
& +\left(1+\frac{1}{r}\right)^{m-1 / 2} \sum_{j=0}^{\infty} \frac{\left|X_{m}\left((t+s)_{r+j+1}\right)-X_{m}\left((t+s)_{r+j}\right)\right|}{(t+s)_{r+j+1}^{m-1 / 2}} \\
& +\sum_{j=0}^{\infty} \frac{\left|X_{m}\left(t_{r+j+1}\right)-X_{m}\left(t_{r+j}\right)\right|}{t_{r+j+1}^{m-1 / 2}} .
\end{aligned}
$$

For the first term of the right hand side of (2.3), by (1.4) we have

$$
E\left(\frac{X_{m}\left((t+s)_{r}\right)-X_{m}\left(t_{r}\right)}{t_{r}^{m-1 / 2}}\right)^{2} \leq\left(1+\frac{\varepsilon}{4}\right)^{2} b_{m 2}\left(1+2^{-r}\right)^{2} h^{2} \leq\left(1+\frac{\varepsilon}{3}\right)^{2} b_{m 2} h^{2},
$$

provided $r=r(\varepsilon)$ is large enough. Hence, noting that the number of points lying within the grid $[0, h] \times[r h, 1]$ with step $h / 2^{r}$ is less than $2^{2 r} / h$, we obtain

$$
\begin{aligned}
& P\left\{\sup _{r h<t \leq 1-h} \sup _{0 \leq s \leq h} \frac{\left|X_{m}\left((t+s)_{r}\right)-X_{m}\left(t_{r}\right)\right|}{t_{r}^{m-1 / 2}} \geq\left(1+\frac{\varepsilon}{3}\right) b_{m 2}^{1 / 2} h x\right\} \\
& \quad \leq \frac{2^{2 r}}{h} \sup _{r h<t \leq 1-h} \sup _{0 \leq s \leq h} P\left\{\frac{\left|X_{m}\left((t+s)_{r}\right)-X_{m}\left(t_{r}\right)\right|}{t_{r}^{m-1 / 2}} \geq\left(1+\frac{\varepsilon}{3}\right) b_{m 2}^{1 / 2} h x\right\} \\
& \quad \leq \frac{2^{2 r}}{h} e^{-x^{2} / 2}
\end{aligned}
$$

by recalling the well-known inequality $1-\Phi(x) \leq(1 / \sqrt{2 \pi} x) e^{-x^{2} / 2}$. (Without loss of generality, assume that $x_{0} \geq 1 / \sqrt{2 \pi}$.)

Consider the second term of the right hand side of (2.3). Note the following inequality:

$$
\begin{aligned}
P\left\{\sup _{i \in I} \sum_{j=0}^{\infty} X_{i j} \geq \sum_{j=0}^{\infty} x_{j}\right\} & \leq \sum_{i \in I} P\left\{\sum_{j=0}^{\infty} X_{i j} \geq \sum_{j=0}^{\infty} x_{j}\right\} \\
& \leq \#(I) \sup _{i \in I} P\left\{\exists j \geq 0: X_{i j} \geq x_{j}\right\} \\
& \leq \#(I) \sup _{i \in I} \sum_{j=0}^{\infty} P\left\{X_{i j} \geq x_{j}\right\}
\end{aligned}
$$


where $X_{i j}, i \in I, j=0,1, \ldots$, are random variables and $x_{j}, j=0,1, \ldots$, are real numbers. Moreover, by (1.4) again, we have

$$
E\left(\frac{X_{m}\left(t_{r+j+1}\right)-X_{m}\left(t_{r+j}\right)}{t_{r+j+1}^{m-1 / 2}}\right)^{2} \leq 2 b_{m 2} h^{2} / 2^{2(r+j+1)}
$$

for any $0<t \leq 1$, provided $r$ is large enough. Furthermore, we may demand

$$
\sqrt{2} \sum_{j=0}^{\infty} 2^{-(r+j+1) / 2} \leq\left(1+\frac{1}{r}\right)^{-m+1 / 2} \frac{\varepsilon}{3} \text {. }
$$

Then we have

$$
\begin{aligned}
P\left\{\sup _{r h<t \leq 1-h}\right. & \left.\sup _{0 \leq s \leq h} \sum_{j=0}^{\infty} \frac{\left|X_{m}\left((t+s)_{r+j+1}\right)-X_{m}\left((t+s)_{r+j}\right)\right|}{(t+s)_{r+j+1}^{m-1 / 2}} \geq\left(1+\frac{1}{r}\right)^{-m+1 / 2} \frac{\varepsilon}{3} b_{m 2}^{1 / 2} h x\right\} \\
& \leq P\left\{\sup _{r h<t \leq 1} \sum_{j=0}^{\infty} \frac{\left|X_{m}\left(t_{r+j+1}\right)-X_{m}\left(t_{r+j}\right)\right|}{t_{r+j+1}^{m-1 / 2}} \geq \sum_{j=0}^{\infty} \sqrt{2} b_{m 2}^{1 / 2}\left(\frac{h}{2^{(r+j+1) / 2}}\right) x\right\} \\
& \leq \frac{2^{r}}{h} \sum_{j=0}^{\infty} e^{-2^{r+j+1} x^{2} / 2} \leq \frac{2^{r}}{h} e^{-x^{2} / 2}
\end{aligned}
$$

for large $r$. Similarly, for the third term of the right hand side of (2.3) we have

$$
P\left\{\sup _{r h<t \leq 1-h} \sup _{0 \leq s \leq h} \sum_{j=0}^{\infty} \frac{\left|X_{m}\left(t_{r+j+1}\right)-X_{m}\left(t_{r+j}\right)\right|}{t_{r+j+1}^{m-1 / 2}} \geq \frac{\varepsilon}{3} b_{m 2}^{1 / 2} h x\right\} \leq \frac{2^{r}}{h} e^{-x^{2} / 2} .
$$

Combining (2.3) $-(2.6)$ we obtain

$$
P\left\{I_{2} \geq(1+\varepsilon) b_{m 2}^{1 / 2} h x\right\} \leq\left(2^{2 r}+2^{r+1}\right) \frac{1}{h} e^{-x^{2} / 2} .
$$

(2.2) and (2.7) together imply the conclusion of Proposition 2.1.

An analogue of Proposition 2.1 in the large increment case is the following.

PROPOSITION 2.2. Let $a_{T}$ be a function of $T$ with $0<a_{T} \leq T$ and $a_{T} / T \rightarrow 0$ as $T \rightarrow \infty$. Then for any $\varepsilon>0$, there exist positive numbers $T_{0}, x_{1}, c_{2}$ and $C_{2}$ such that for any $T \geq T_{0}$ and $x \geq x_{1}$,

$P\left\{\sup _{0<t \leq T-a_{T}} \sup _{0 \leq s \leq a_{T}} \frac{\left|X_{m}(t+s)-X_{m}(t)\right|}{\left(t \vee a_{T}\right)^{m-1 / 2}} \geq(1+\varepsilon) b_{m 2}^{1 / 2} a_{T} x\right\} \leq C_{2}\left(e^{-c_{2} x^{2}}+T a_{T}^{-1} e^{-x^{2} / 2}\right)$.

The proof is similar to that of Proposition 2.1, and hence, is omitted. 


\section{Moduli of continuity}

We need another well-known lemma.

LEMMA 3.1 (Slepian). Let $G(t)$ and $G^{*}(t)$ be Gaussian processes on $[0, T]$ for some $0<T<\infty$, possessing continuous sample path functions with $E G(t)=$ $E G^{*}(t)=0, E G^{2}(t)=E G^{* 2}(t)=1$, and let $\rho(s, t)$ and $\rho^{*}(s, t)$ be their respective covariance functions. Suppose that we have $\rho(s, t) \geq \rho^{*}(s, t), s, t \in[0, T]$. Then for any real $u$,

$$
P\left\{\sup _{0 \leq t \leq T} G(t) \leq u\right\} \geq P\left\{\sup _{0 \leq t \leq T} G^{*}(t) \leq u\right\}
$$

Put $\log x=\ln (e \vee x)$.

\section{THEOREM 3.1.}

(3.1) $\quad \limsup _{h \rightarrow 0} \sup _{0<t \leq 1-h} \sup _{0 \leq s \leq h} \frac{\left|X_{m}(t+s)-X_{m}(t)\right|}{b_{m 2}^{1 / 2}(t \vee h)^{m-1 / 2} h\left(2 \log h^{-1}\right)^{1 / 2}} \leq 1$ almost surely,

$$
\liminf _{h \rightarrow 0} \sup _{0<t \leq 1-h} \frac{\left|X_{m}(t+h)-X_{m}(t)\right|}{b_{m 2}^{1 / 2}(t \vee h)^{m-1 / 2} h\left(2 \log \log h^{-1}\right)^{1 / 2}} \geq 1 \quad \text { almost surely. }
$$

REMARK 3.1. It is interesting to find the exact factors such that equality signs in (3.1) and/or (3.2) hold. For Lévy's moduli of continuity of a Brownian motion $W(\cdot)$, the ' $\left(\log h^{-1}\right)^{1 / 2}$, makes the equality sign in (3.1) hold. For $X_{m}(\cdot)$, there are certain difficulties because its increments are neither independent nor stationary.

ProOF. First we prove (3.1). For any given $\varepsilon>0$, by Propositon 2.1, there exist $c_{1}=c_{1}(\varepsilon)>0$ and $C_{1}=C_{1}(\varepsilon)>0$ such that

$$
\begin{aligned}
P\left\{\sup _{0<t \leq 1-h} \sup _{0 \leq s \leq h} \frac{\left|X_{m}(t+s)-X_{m}(t)\right|}{b_{m 2}^{1 / 2}(t \vee h)^{m-1 / 2} h\left(2 \log h^{-1}\right)^{1 / 2}} \geq(1+\varepsilon)^{2}\right\} \\
\quad \leq C_{1}\left(\exp \left\{-2 c_{1}(1+\varepsilon)^{2} \log h^{-1}\right\}+h^{-1} \exp \left\{-(1+\varepsilon)^{2} \log h^{-1}\right\}\right) \\
\quad \leq C_{1}\left(h^{2 c_{1}}+h^{2 \varepsilon}\right) .
\end{aligned}
$$

Taking $h_{n}=n^{-A}$ with $A>\left(2\left(\varepsilon \wedge c_{1}\right)\right)^{-1}$, we obtain

$$
\sum_{n=1}^{\infty} P\left\{\sup _{0<t \leq 1-h_{n}} \sup _{0 \leq s \leq h_{n}} \frac{\left|X_{m}(t+s)-X_{m}(t)\right|}{b_{m 2}^{1 / 2}\left(t \vee h_{n}\right)^{m-1 / 2} h_{n}\left(2 \log h_{n}^{-1}\right)^{1 / 2}} \geq(1+\varepsilon)^{2}\right\}<\infty,
$$


which, in combination with the Borel-Cantelli lemma, implies

$$
\limsup \sup _{n \rightarrow \infty} \sup _{0<t \leq 1-h_{n}} \frac{\left|X_{m}(t+s)-X_{m}(t)\right|}{b_{m 2}^{1 / 2}\left(t \vee h_{n}\right)^{m-1 / 2} h_{n}\left(2 \log h_{n}^{-1}\right)^{1 / 2}} \leq(1+\varepsilon)^{2}
$$

The procedure from (3.3) to (3.1) is routine, and hence, is omitted.

Next we show (3.2). Let $h_{n}=n^{-A_{n}}$ with $A_{n}=n^{(\log \log n)^{-1}} \uparrow \infty$ as $n \rightarrow \infty$. Define

$$
Y(i)=\frac{X_{m}\left((i+1) h_{n}\right)-X_{m}\left(i h_{n}\right)}{\left(i h_{n}\right)^{m-1 / 2}}, \quad 0<i \leq n^{A_{n}}-1 .
$$

By (1.4), $E Y(i)^{2} \geq b_{m 2} h_{n}^{2}$. We have that, for $i \leq j$,

(3.4) $E(Y(i) Y(j))$

$$
\begin{aligned}
= & \frac{1}{(m !)^{2}\left(i h_{n}\right)^{m-1 / 2}\left(j h_{n}\right)^{m-1 / 2}}\left\{\int_{0}^{(i+1) h_{n}}\left((i+1) h_{n}-s\right)^{m}\left((j+1) h_{n}-s\right)^{m} d s\right. \\
& -\int_{0}^{(i+1) h_{n}}\left((i+1) h_{n}-s\right)^{m}\left(j h_{n}-s\right)^{m} d s \\
& \left.-\int_{0}^{i h_{n}}\left(i h_{n}-s\right)^{m}\left((j+1) h_{n}-s\right)^{m} d s+\int_{0}^{i h_{n}}\left(i h_{n}-s\right)^{m}\left(j h_{n}-s\right)^{m} d s\right\} \\
= & h_{n}^{2} \sum_{p=0}^{m} \sum_{q=0}^{m}\left(\begin{array}{l}
m \\
p
\end{array}\right)\left(\begin{array}{l}
m \\
q
\end{array}\right) \frac{1}{(m !)^{2}(2 m-p-q+1)(i j)^{m-1 / 2}} \\
& \times\left\{(i+1)^{2 m-q+1}(j+1)^{q}-(i+1)^{2 m-q+1} j^{q}-i^{2 m-q+1}(j+1)^{q}+i^{2 m-q+1} j^{q}\right\} \\
= & h_{n}^{2} \sum_{p=0}^{m} \sum_{q=0}^{m}\left(\begin{array}{c}
m \\
p
\end{array}\right)\left(\begin{array}{l}
m \\
q
\end{array}\right) \frac{\left((i+1)^{2 m-q+1}-i^{2 m-q+1}\right)\left((j+1)^{q}-j^{q}\right)}{(m !)^{2}(2 m-p-q+1)(i j)^{m-1 / 2}} \\
= & h_{n}^{2} \sum_{p=0}^{m} \sum_{q=0}^{m}\left(\begin{array}{c}
m \\
p
\end{array}\right)\left(\begin{array}{c}
m \\
q
\end{array}\right) \frac{4(2 m-q+1) q(i / j)^{m-q+1 / 2}}{(m !)^{2}(2 m-p-q+1)}(1+O(1 / i)) .
\end{aligned}
$$

Let $n_{1}=\left[A_{n} \log n\right], Z(i)=Y\left(e^{i}\right), i=0,1, \ldots, n_{1}$,

$$
c_{m}=\sum_{p=0}^{m} \sum_{q=0}^{m}\left(\begin{array}{l}
m \\
p
\end{array}\right)\left(\begin{array}{l}
m \\
q
\end{array}\right) \frac{4(2 m-q+1) q}{(m !)^{2}(2 m-p-q+1)},
$$

and $D_{n}=3 \log \log n$. (3.4) implies that for $i \geq n_{1} / 3$ and $j-i \geq D_{n}$,

$$
E(Z(i) Z(j)) \leq h_{n}^{2} c_{m} e^{-(j-i) / 2}(1+O(1 / i)) \leq c_{m}(\log n)^{-1} h_{n}^{2},
$$

provided $n$ is large enough. Let $\left\{\xi_{i}, i \geq 0\right\}$ and $\zeta$ be independent normal random variables with means zero and $E \xi_{i}^{2}=E Z(i)^{2}-c_{m}(\log n)^{-1} h_{n}^{2}=(1+o(1)) b_{m 2} h_{n}^{2}$ as $n \rightarrow \infty$ (recalling (1.4)), $E \zeta^{2}=c_{m}(\log n)^{-1} h_{n}^{2}$. Define $\gamma_{i}=\xi_{i}+\zeta$. Then 
$E \gamma_{i}^{2}=E Z(i)^{2}$ and $E Z(i) Z(j) \leq E \gamma_{i} \gamma_{j}$. Let $I=\left\{i: n_{1} / 3 \leq i \leq n_{1}-1, i\right.$ $\left.\bmod D_{n}\right\}$, then $\#(I) \geq n_{1} /\left(2 D_{n}\right)$ for large $n$. Hence by Slepian's lemma and using the well-known inequality

$$
1-\Phi(x) \geq \frac{1}{\sqrt{2 \pi}}\left(\frac{1}{x}-\frac{1}{x^{3}}\right) e^{-x^{2} / 2},
$$

we obtain that for large $n$

$$
\text { (3.6) } \begin{aligned}
& P\left\{\max _{\substack{n_{1} / 3 \leq i \leq n_{1}-1 \\
i \\
\bmod D_{n}}} Z(i) \leq(1-\varepsilon) b_{m 2}^{1 / 2} h_{n}\left(2 \log \log h_{n}^{-1}\right)^{1 / 2}\right\} \\
& \leq P\left\{\max _{\substack{n_{1} / 3 \leq i \leq n_{1}-1 \\
i}} \gamma_{i} \leq(1-\varepsilon) b_{m 2}^{1 / 2} h_{n}\left(2 \log \log h_{n}^{-1}\right)^{1 / 2}\right\} \\
& \leq P\left\{\max _{\substack{n_{1} / 3 \leq i \leq n_{1}-1 \\
i}} \xi_{i} \leq\left(1-\frac{\varepsilon}{2}\right) b_{m 2}^{1 / 2} h_{n}\left(2 \log \log h_{n}^{-1}\right)^{1 / 2}\right\} \\
&+P\left\{\zeta \geq \frac{\varepsilon}{2} b_{m 2}^{1 / 2} h_{n}\left(2 \log \log h_{n}^{-1}\right)^{1 / 2}\right\} \\
& \leq\left(1-P\left\{\xi_{i}>\left(1-\frac{\varepsilon}{2}\right) b_{m 2}^{1 / 2} h_{n}\left(2 \log \log h_{n}^{-1}\right)^{1 / 2}\right\}\right)^{n_{1} /\left(2 D_{n}\right)} \\
&+\exp \left\{-\frac{\varepsilon^{2} b_{m 2}}{4 c_{m}(\log n)^{-1}} \log \log h_{n}^{-1}\right\} \\
& \leq\left(1-\frac{1}{\left(8 \pi \log \log h_{n}^{-1}\right)^{1 / 2}} \exp \left\{-\left(1-\frac{\varepsilon}{2}\right) \log \log h_{n}^{-1}\right\}\right)^{n_{1} /\left(2 D_{n}\right)}+n^{-2} \\
&=\left(1-\frac{\left(\log h_{n}^{-1}\right)^{(1-\varepsilon / 2)}}{\left(8 \pi \log \log h_{n}^{-1}\right)^{1 / 2}}\right)^{n_{1} /\left(2 D_{n}\right)}+n^{-2} \\
& \leq \exp \left\{-\frac{\left(\log h_{n}^{-1}\right)^{-(1-\varepsilon / 2)} n_{1}}{\left.2 D_{n}\left(8 \pi \log \log h_{n}^{-1}\right)^{1 / 2}\right\}+n^{-2} \leq 2 n^{-2}} .\right.
\end{aligned}
$$

Inequality (3.6) implies

$$
\sum_{n=1}^{\infty} P\left\{\max _{0 \leq i \leq n_{1}-1} Z(i) \leq(1-\varepsilon) b_{m 2}^{1 / 2} h_{n}\left(2 \log \log h_{n}^{-1}\right)^{1 / 2}\right\}<\infty,
$$

and by the Borel-Cantelli lemma it follows that

$$
\liminf _{n \rightarrow \infty} \max _{0 \leq i \leq n_{1}-1} \frac{Z(i)}{b_{m 2}^{1 / 2} h_{n}\left(2 \log \log h_{n}^{-1}\right)^{1 / 2}} \geq 1-\varepsilon \quad \text { a.s. }
$$


And hence we conclude

$$
\liminf _{n \rightarrow \infty} \sup _{h_{n} \leq t \leq 1-h_{n}} \frac{X_{m}\left(t+h_{n}\right)-X_{m}(t)}{b_{m 2}^{1 / 2} t^{m-1 / 2} h_{n}\left(2 \log \log h_{n}^{-1}\right)^{1 / 2}} \geq 1-\varepsilon \quad \text { a.s. }
$$

Considering $h_{n+1}<h \leq h_{n}$, we have

$$
\begin{aligned}
\sup _{0<t \leq 1-h} & \frac{\left|X_{m}(t+h)-X_{m}(t)\right|}{b_{m 2}^{1 / 2}(t \vee h)^{m-1 / 2} h\left(2 \log \log h^{-1}\right)^{1 / 2}} \\
\geq & \sup _{h_{n} \leq t \leq 1-h} \frac{\left|X_{m}\left(t+h_{n}\right)-X_{m}(t)+X_{m}(t+h)-X_{m}\left(t+h_{n}\right)\right|}{b_{m 2}^{1 / 2} t^{m-1 / 2} h\left(2 \log \log h^{-1}\right)^{1 / 2}} \\
\geq & \sup _{h_{n} \leq t \leq 1-h_{n}} \frac{\left|X_{m}\left(t+h_{n}\right)-X_{m}(t)\right|}{b_{m 2}^{1 / 2} t^{m-1 / 2} h_{n}\left(2 \log \log h_{n}^{-1}\right)^{1 / 2}} \\
& -2 \sup _{h_{n}<t \leq 1-\left(h_{n}-h_{n+1}\right)} \sup _{0 \leq s \leq h_{n}-h_{n+1}} \frac{\left|X_{m}\left(t+h_{n+1}+s\right)-X_{m}\left(t+h_{n+1}\right)\right|}{b_{m 2}^{1 / 2}\left(t+h_{n+1}\right)^{m-1 / 2}\left(h_{n}-h_{n+1}\right)} \\
& \times \frac{\left(t+h_{n+1}\right)^{m-1 / 2}\left(h_{n}-h_{n+1}\right)\left(\log \left(h_{n}-h_{n+1}\right)^{-1}\right)^{1 / 2}}{\left(2 \log \left(h_{n}-h_{n+1}\right)^{-1}\right)^{1 / 2} t^{m-1 / 2} h_{n+1}\left(\log \log h_{n+1}^{-1}\right)^{1 / 2}} .
\end{aligned}
$$

By the derivative calculus for the function $f(x)=x^{-A_{x}}$, we have

$$
h_{n}-h_{n+1}=h_{n+1} \frac{A_{n} \log n}{n \log \log n}(1+o(1)) .
$$

Therefore,

$$
\lim _{n \rightarrow \infty} \sup _{h_{n}<t \leq 1-\left(h_{n}-h_{n+1}\right)} \frac{\left(t+h_{n+1}\right)^{m-1 / 2}\left(h_{n}-h_{n+1}\right)\left(\log \left(h_{n}-h_{n+1}\right)^{-1}\right)^{1 / 2}}{t^{m-1 / 2} h_{n+1}\left(\log \log h_{n+1}^{-1}\right)^{1 / 2}}=0 .
$$

Consequently we conclude (3.2) by (3.8), (3.9) and (3.1). This completes the proof of Theorem 3.1.

\section{Large increments}

THEOREM 4.1. Let $a_{T}$ be a continuous function of $T$ with $0<a_{T} \leq T$ and suppose that

$$
\lim _{n \rightarrow \infty} \frac{\sup _{n-1<t \leq n} a_{t}}{\inf _{n-1<t \leq n} a_{t}}=1
$$

and

$$
\lim _{T \rightarrow \infty} \log \left(T / a_{T}\right) / \log \log T=\infty
$$

\section{Then}

$$
\limsup _{T \rightarrow \infty} \sup _{0<r \leq T-a_{T}} \sup _{0 \leq s \leq a_{T}} \frac{\left|X_{m}(t+s)-X_{m}(t)\right|}{b_{m 2}^{i / 2}\left(t \vee a_{T}\right)^{m-1 / 2} a_{T}\left(2 \log \left(T / a_{T}\right)\right)^{1 / 2}} \leq 1 \quad \text { a.s. }
$$


If, instead of (4.2), for any $\varepsilon>0$ there exists $T_{0}>0$ such that for $T>T_{0}$

$$
\begin{aligned}
& \left(\log \frac{T}{a_{T}}\right)^{(\log \log \log T)^{1 / \varepsilon}} \geq \log T, \\
& \left(\log a_{T}\right)^{2(1-\varepsilon) \log \log \log a_{T}} \geq \log T,
\end{aligned}
$$

then

$$
\liminf _{T \rightarrow \infty} \sup _{0<t \leq T-a_{T}} \frac{\left|X_{m}\left(t+a_{T}\right)-X_{m}(t)\right|}{b_{m 2}^{1 / 2}\left(t \vee a_{T}\right)^{m-1 / 2} a_{T}\left(2 \log \log \left(T / a_{T}\right)\right)^{1 / 2}} \geq 1 \quad \text { a.s. }
$$

Proof. First we prove (4.3). Let $\theta>1$ and for integers $k$ and $j$ let

$$
A_{k j}=\left\{T: \theta^{k-1}<T \leq \theta^{k}, \theta^{j-1}<a_{T} \leq \theta^{j}\right\}
$$

In the sequel, we always consider $k$ and $j$ such that $A_{k j}$ is non-empty. For any $\boldsymbol{A}>0$, by condition (4.2), there exists $k_{0}$ such that for $k \geq k_{0}$

$$
\log \theta^{k-j} / \log \log \theta^{k} \geq A \text {, }
$$

that is,

$$
j \leq k-\left[(A / \log \theta) \log k+\theta_{1}\right]=: k_{1},
$$

where $\theta_{1}=A(\log \log \theta) / \log \theta$. Then, noting that $b_{m 2}^{1 / 2}\left(t \vee a_{T}\right)^{m-1 / 2} a_{T}\left(2 \log \left(T / a_{T}\right)\right)^{1 / 2}$ is an increasing function of both $T$ and $a_{T}$, we have

(4.9) $\limsup _{T \rightarrow \infty} \sup _{0<t \leq T-a_{T}} \sup _{0 \leq s \leq a_{T}} \frac{\left|X_{m}(t+s)-X_{m}(t)\right|}{b_{m 2}^{1 / 2}\left(t \vee a_{T}\right)^{m-1 / 2} a_{T}\left(2 \log \left(T / a_{T}\right)\right)^{1 / 2}}$

$$
\begin{aligned}
& \leq \limsup _{k \rightarrow \infty} \sup _{-\infty<j \leq k_{1}} \sup _{0<t \leq \theta^{k}-\theta^{j-1}} \sup _{0 \leq s \leq \theta^{j}} \frac{\left|X_{m}(t+s)-X_{m}(t)\right|}{b_{m 2}^{1 / 2}\left(t \vee \theta^{j-1}\right)^{m-1 / 2} \theta^{j-1}\left(2 \log \theta^{k-j}\right)^{1 / 2}} \\
& \leq \limsup \sup _{k \rightarrow \infty} \sup _{-\infty<j \leq k_{1}} \sup _{0<t \leq \theta^{k+1}-\theta^{j}} \frac{\theta^{m+1 / 2}\left|X_{m}(t+s)-\theta_{m}(t)\right|}{b_{m 2}^{1 / 2}\left(t \vee \theta^{j}\right)^{m-1 / 2} \theta^{j}\left(2 \log \theta^{k-j}\right)^{1 / 2}} .
\end{aligned}
$$

Using Proposition 2.2 and (4.8) we have

$$
\begin{aligned}
& P\left\{\sup _{-\infty<j \leq k_{1}} \sup _{0<t \leq \theta^{k+1}-\theta^{j}} \sup _{0 \leq s \leq \theta^{j}} \frac{\left|X_{m}(t+s)-X_{m}(t)\right|}{b_{m 2}^{1 / 2}\left(t \vee \theta^{j}\right)^{m-1 / 2} \theta^{j}\left(2 \log \theta^{k-j}\right)^{1 / 2}} \geq(1+\varepsilon)^{2}\right\} \\
& \quad \leq C_{2} \sum_{j=-\infty}^{k_{1}}\left(\exp \left\{-2 c_{2}(1+\varepsilon)^{2} \log \theta^{k-j}\right\}+\theta^{k-j+1} \exp \left\{-(1+\varepsilon)^{2} \log \theta^{k-j}\right\}\right) \\
& \quad \leq C_{2} \sum_{j=-\infty}^{k_{1}}\left(\theta^{-2 c_{2}(1+\varepsilon)^{2}(k-j)}+\theta^{-2 \varepsilon(k-j)+1}\right) \\
& \leq c\left(\theta^{-2 c_{2}(1+\varepsilon)^{2}\left[(A / \log \theta) \log k+\theta_{1}\right]}+\theta^{-2 \varepsilon\left[(A / \log \theta) \log k+\theta_{1}\right]+1}\right) \leq c k^{-2}
\end{aligned}
$$


for some $c>0$ by taking $A=(\log \theta) /\left(c_{2}(1+\varepsilon)^{2} \wedge \varepsilon\right)$. Hence, from the Borel-Cantelli lemma we obtain

$$
\limsup \sup _{k \rightarrow \infty} \sup _{-\infty<j \leq k_{1}} \sup _{0 \leq t \leq \theta^{k+1}-\theta^{j}} \frac{\left|X_{m}(t+s)-X_{m}(t)\right|}{0 \leq \theta^{j}} \leq(1+\varepsilon)^{2} \quad \text { a.s. }
$$

which, in combination with (4.9), implies (4.3) by arbitrariness of $\theta>1$.

Next we show (4.6). Let $A_{j}=j^{(\log \log j)^{-1}}$ again, and let $B_{0}=0, B_{j}=j^{A_{j}}$, $j=1,2, \ldots, C_{k j}=\left\{T: B_{k-1}<T \leq B_{k}, B_{j-1}<a_{T} \leq B_{j}\right\}$. By condition (4.4), for any $A>0$, there exists an integer $j_{0}$ such that for $j \geq j_{0}$

$$
\log \left(B_{k} / B_{j}\right) \geq\left(\log B_{k}\right)^{(\log \log k)^{-1}} \geq A_{k}^{(\log \log k)^{-A}} .
$$

On the other hand, by the derivative calculus for the function $g(x)=\log B_{x}$, we have

$$
\log B_{k}-\log B_{j} \leq 2(k-j) \frac{A_{k} \log k}{k \log \log k}
$$

which, in combination with (4.10), implies that

$$
j \leq k-\left[\frac{k \log \log k}{2 \log k} A_{k}^{-1+(\log \log k)^{-1}}\right]=: k_{2} \text {. }
$$

Noting that $b_{m 2}^{1 / 2}\left(t \vee a_{T}\right)^{m-1 / 2} a_{T}\left(2 \log \log \left(T / a_{T}\right)\right)^{1 / 2}$ is an increasing function of both $T$ and $a_{T}$ we can write

(4.11) $\liminf _{T \rightarrow \infty} \sup _{0<t \leq T-a_{T}} \frac{\left|X_{m}\left(t+a_{T}\right)-X_{m}(t)\right|}{b_{m 2}^{1 / 2}\left(t \vee a_{T}\right)^{m-1 / 2} a_{T}\left(2 \log \log \left(T / a_{T}\right)\right)^{1 / 2}}$

$$
\begin{aligned}
\geq & \liminf _{k \rightarrow \infty} \inf _{1 \leq j \leq k_{2}} \inf _{T \in C_{k j}} \sup _{0<t \leq T-a_{T}} \frac{\left|X_{m}\left(t+a_{T}\right)-X_{m}(t)\right|}{b_{m 2}^{1 / 2}\left(t \vee a_{T}\right)^{m-1 / 2} a_{T}\left(2 \log \log \left(T / a_{T}\right)\right)^{1 / 2}} \\
\geq & \liminf _{k \rightarrow \infty} \inf _{1 \leq j \leq k_{2}} \sup _{0<t \leq B_{k-1} / 2} \frac{\left|X_{m}\left(t+B_{j}\right)-X_{m}(t)\right|}{b_{m 2}^{1 / 2}\left(t \vee B_{j}\right)^{m-1 / 2} B_{j}\left(2 \log \log \left(B_{k} / B_{j}\right)\right)^{1 / 2}} \\
- & \limsup _{k \rightarrow \infty} \sup _{0<t \leq B_{k}-\left(B_{j}-B_{j-1}\right)} \sup _{0 \leq s \leq B_{j}-B_{j-1}} \frac{\left|X_{m}(t+s)-X_{m}(t)\right|}{b_{m 2}^{1 / 2}\left(t \vee\left(B_{j}-B_{j-1}\right)\right)^{m-1 / 2}\left(B_{j}-B_{j-1}\right)} \\
& \times \frac{\left(t \vee\left(B_{j}-B_{j-1}\right)\right)^{m-1 / 2}\left(B_{j}-B_{j-1}\right)\left(\log \left(B_{k} /\left(B_{j}-B_{j-1}\right)\right)\right)^{1 / 2}}{\left(2 \log \left(B_{k} /\left(B_{j}-B_{j-1}\right)\right)\right)^{1 / 2}\left(t \vee B_{j}\right)^{m-1 / 2} B_{j}\left(\log \log \left(B_{k} / B_{j}\right)\right)^{1 / 2}} \\
= & : J_{1}-J_{2} .
\end{aligned}
$$

By the derivative calculus for the function $h(x)=B_{x}$, we have

$$
\frac{B_{j}-B_{j-1}}{B_{j}} \leq \frac{2 A_{j} \log j}{j \log \log j}
$$


The last inequality and condition (4.5) imply that, as $k \rightarrow \infty$,

$$
\log B_{k} \leq(1+o(1)) \log B_{k-1} \leq 2\left(\log B_{j}\right)^{2(1-\varepsilon) \log \log \log B_{j}} \leq 2\left(A_{j} \log j\right)^{2(1-\varepsilon) \log \log j} .
$$

Hence

$$
\begin{gathered}
\frac{\left(t \vee\left(B_{j}-B_{j-1}\right)\right)^{m-1 / 2}\left(B_{j}-B_{j-1}\right)\left(\log \left(B_{k} /\left(B_{j}-B_{j-1}\right)\right)\right)^{1 / 2}}{\left(t \vee B_{j}\right)^{m-1 / 2} B_{j}\left(\log \log \left(B_{k} / B_{j}\right)\right)^{1 / 2}} \\
\quad \leq \frac{B_{j}-B_{j-1}}{B_{j}}\left(\log B_{k}\right)^{1 / 2} \leq \frac{2 \sqrt{2} A_{j} \log j}{j \log \log j} \cdot\left(A_{j} \log j\right)^{(1-\varepsilon) \log \log j} \\
\quad=\frac{2 \sqrt{2} A_{j}(\log j)^{1+(1-\varepsilon) \log \log j}}{j^{\varepsilon} \log \log j} \rightarrow 0 \text { as } j \rightarrow \infty .
\end{gathered}
$$

Then by (4.3) and (4.12) we obtain

$$
J_{2}=0 \quad \text { a.s. }
$$

Consider $J_{1}$ and for fixed $k$, define

$$
Y_{j}(i)=\frac{X_{m}\left((i+1) B_{j}\right)-X_{m}\left(i B_{j}\right)}{\left(i B_{j}\right)^{m-1 / 2}}, \quad 0<i \leq B_{k} / B_{j}-1, j=0,1, \ldots, k_{2} .
$$

Furthermore, let $Z_{j}(i)=Y_{j}\left(e^{i}\right), i=0,1, \ldots, k_{3}-1$ with $k_{3}=\left[\log \left(B_{k} / B_{j}\right)\right]$. Similarly to (3.5), we have

$$
E Z_{j}\left(i_{1}\right) Z_{j}\left(i_{2}\right) \leq c_{m}^{\prime}(\log \log k)^{-A-2} B_{j}^{2}
$$

for some $c_{m}^{\prime}>0$ and any $i_{1} \geq k_{3} / 3, i_{2}-i_{1} \geq D_{k}^{\prime}:=3(A+2) \log \log \log k$. Let $\left\{\xi_{i j}, i \geq 0\right\}$ and $\zeta_{j}$ be independent normal random variables with means zero and $E \xi_{i j}^{2}=E Z_{j}(i)^{2}-c_{m}^{\prime}(\log \log k)^{-A-2} B_{j}^{2}, E \zeta_{j}^{2}=c_{m}^{\prime}(\log \log k)^{-A-2} B_{j}^{2}$. Then, similarly to (3.6), using (4.10) with $A>6 / \varepsilon$ we obtain for all large $k$

$$
\begin{aligned}
& P\left\{\inf _{\substack{0 \leq j \leq k_{2} k_{3} / 3 \leq i \leq k_{3}-1 \\
i}} Z_{j}(i) \leq(1-\varepsilon) b_{m 2}^{1 / 2} B_{j}\left(2 \log \log \left(B_{k} / B_{j}\right)\right)^{1 / 2}\right\} \\
& \leq \sum_{j=0}^{k_{2}}\left(\exp \left\{-\frac{\left(\log \left(B_{k} / B_{j}\right)\right)^{-(1-\varepsilon / 2)} k_{3}}{2 D_{k}^{\prime}\left(8 \pi \log \log \left(B_{k} / B_{j}\right)\right)^{1 / 2}}\right\}\right. \\
&\left.+\exp \left\{-\frac{\varepsilon^{2} b_{m 2}}{4 c_{m}^{\prime}(\log \log k)^{-A-2}} \log \log \left(B_{k} / B_{j}\right)\right\}\right) \\
& \leq c \sum_{j=0}^{k_{2}}\left(\exp \left\{-\frac{\left(\log \left(B_{k} / B_{j}\right)\right)^{\varepsilon / 2}}{D_{k}^{\prime}\left(8 \pi \log \log \left(B_{k} / B_{j}\right)\right)^{1 / 2}}\right\}\right.
\end{aligned}
$$




$$
\left.+\exp \left\{-\frac{\varepsilon^{2} b_{m 2}}{4 c_{m}^{\prime}}(\log \log k) \log k\right\}\right) \text {. }
$$

It is easy to see that

$$
D_{k}^{\prime}=o\left(\log \left(B_{k} / B_{j}\right)\right), \quad \log \log \left(B_{k} / B_{j}\right)=o\left(\log \left(B_{k} / B_{j}\right)\right)
$$

So for large $k$,

$$
\exp \left\{-\frac{\left(\log \left(B_{k} / B_{j}\right)\right)^{\varepsilon / 2}}{D_{k}^{\prime}\left(8 \pi \log \log \left(B_{k} / B_{j}\right)\right)^{1 / 2}}\right\} \leq \exp \left\{-\left(\log \left(B_{k} / B_{j}\right)\right)^{\varepsilon / 3}\right\}
$$

Combining it with (4.14) implies

$$
\sum_{k=1}^{\infty} P\left\{\inf _{0 \leq j \leq k_{2}} \max _{0 \leq i \leq k_{3}-1} Z_{j}(i) \leq(1-\varepsilon) b_{m 2}^{1 / 2} B_{j}\left(2 \log \log \left(B_{k} / B_{j}\right)\right)^{1 / 2}\right\}<\infty
$$

Hence

$$
J_{1} \geq 1-\varepsilon \quad \text { a.s. }
$$

Combining (4.15) with (4.13) we conclude that (4.4) holds. This completes the proof of Theorem 4.1.

\section{Acknowledgements}

The author would like to thank the referee for valuable suggestions. The project was supported by NSFC (19571021) and NSFZP (199016).

\section{References}

[1] M. Csörgó and P. Révész, Strong approximations in probability and statistics (Academic Press, New York, 1981).

[2] A. Lachal, 'Local asymptotic classes for the successive primitives of Brownian motion', Ann. Probab. 25 (1997), 1712-1734.

[3] — , 'Regular points for the successive primitives of Brownian motion', J. Math. Kyoto Univ. 37 (1997), 99-119.

[4] L. A. Shepp, 'Radon-Nikodym derivatives of Gaussian measures', Ann. Math. Statist. 37 (1966), 321-354.

[5] G. Wahba, 'Improper priors, spline smoothing and the problem of guarding against model error in regression', J. Roy. Statist. Soc. Ser. B 40 (1978), 364-372.

[6] _ , 'Bayesian 'confidence intervals' for the cross-validated smoothing spline', J. Roy. Statist. Soc. Ser. B 45 (1983), 133-150. 
[7] H. Watanabe, 'An asymptotic property of Gaussian processes I', Trans. Amer. Math. Soc. 148 (1970), 233-248.

Department of Mathematics

Zhejiang University, Xixi Campus

Hangzhou

Zhejiang 310028

P. R. China

e-mail: zlin@mail.hz.zj.cn 